Anales de Geografía de la Universidad Complutense ISSN: 0211-9803

http://dx.doi.org/10.5209/AGUC. 62488

\title{
Los saberes campesinos como estrategia de desarrollo rural en la Serranía de los Yariguíes (Santander, Colombia) ${ }^{1}$
}

\author{
Paulina-Alejandra Vergara-Buitrago ${ }^{2}$ \\ Recibido: 17 de octubre del 2017/ Enviado a evaluar: 14 de enero del 2018/ Aceptado: 18 de octubre del 2018
}

Resumen. El saber propio de las comunidades campesinas, constituye un potencial de cambio para el manejo racional y la protección de los ecosistemas, ya que implica la producción y la vinculación del campesino como actor dentro de su propio territorio, además de la participación del mismo en la conservación del medio ambiente. Se realizó un trabajo participativo local, con el propósito de caracterizar los saberes de los campesinos ubicados en la microcuenca la Cinco Mil, Serranía de los Yariguíes (Santander, Colombia), visibilizando la contribución de estos saberes a la protección y conservación de ecosistemas estratégicos. Los resultados muestran que la recuperación y apropiación de los saberes campesinos, representan estrategias sostenibles frente a las técnicas de producción agrícola actuales, así como acciones para promover el desarrollo rural de la población de la microcuenca la Cinco Mil.

Palabras clave: Comunidad; ecosistemas; saberes campesinos; sostenibilidad; Serranía de los Yariguíes.

\section{[en] Peasant knowledge as a strategy for rural development in the Serranía de los Yariguíes (Santander, Colombia)}

Abstract. The knowledge of peasant communities is a potential for change for the rational management and protection of ecosystems, as it implies the production and linkage of the farmer as an actor within his own territory, in addition to the participation of the same in the conservation of the environment. A local participatory work was carried out, with the purpose of characterizing the knowledge of the peasants

1 Este artículo se ha elaborado en el marco del proyecto "Reconocimiento de los saberes campesinos en la microcuenca la Cinco Mil, Serranía de los Yariguíes (Santander, Colombia), como aporte a la conservación de servicios ecosistémicos" de la Maestría en Geografía Convenio UPTC-IGAC. A la Universidad Pedagógica y Tecnológica de Colombia, a través de la Vicerrectoría de Investigaciones y Extensión, al convenio UptcEcopetrol 5211740 proyecto sobre restauración ecológica en el Parque Nacional Natural Serranía de los Yariguíes (Santander, Colombia). Al Gobierno de México por la beca para Extranjeros 2017, y al Programa de Movilidad Académica entre todas las Instituciones asociadas a la AUIP

2 Universidad Pedagógica y Tecnológica de Colombia, Grupo de Investigación Sistemática Biológica (SisBio).

E-mail: paulina.vergara@uptc.edu.co 
located in the Cinco Mil micro-watershed, Serranía de los Yariguíes (Santander, Colombia), making visible the contribution of this knowledge to the protection and conservation of strategic ecosystems. The results show that the recovery and appropriation of peasant knowledge represent sustainable strategies in the face of current agricultural production techniques, as well as actions to promote the rural development of the population of the Cinco Mil micro-watershed.

Key Words: Community; ecosystems; peasant knowledge; sustainability; Serranía de los Yariguíes.

[fr] La connaissance paysanne en tant que stratégie de développement rural dans la Serranía de los Yariguíes (Santander, Colombie)

Résumé. Le savoir-faire des communautés paysannes constitue un potentiel de changement pour la gestion rationnelle et la protection des écosystèmes puisqu'il implique la production et l'implication du paysan en tant qu'acteur sur son propre territoire ainsi que sa participation à la conservation de l'environnement. Un travail participatif local a été mené dans le but de caractériser la connaissance des paysans du bassin des Cinco Mil, la Serranía de los Yariguíes (Santander, Colombie), en mettant en évidence la contribution de cette connaissance à la protection et à la conservation des écosystèmes stratégiques. Les résultats montrent que la récupération et l'appropriation des connaissances paysannes représentent des stratégies durables face aux techniques de production agricole actuelles, ainsi que des actions visant à promouvoir le développement rural de la population du microbasin hydrographique des Cinco Mil.

Mots Clés: Communauté ; écosystèmes ; savoir paysan ; durabilité ; Serranía de los Yariguíes.

Cómo citar. Vergara-Buitrago, P.A. (2018): Los saberes campesinos como estrategia de desarrollo rural en la Serranía de los Yariguíes (Santander, Colombia). Anales de Geografía de la Universidad Complutense, 38(2), 461-476.

Sumario. 1. Introducción. 1.1. Enclavamiento territorial en territorios periféricos. 2. Metodología. 2.1 Fases de trabajo. 2.2 Área de estudio. 3. Marco referencial sobre saberes campesinos. 4. Resultados y discusión. 5. Conclusiones. 6. Referencias bibliográficas.

\section{Introducción}

\subsection{Enclavamiento territorial en territorios periféricos}

La historia social del espacio territorial que hoy se percibe en Santander, trasciende varios miles de años. De hecho, la forma como los antepasados estaban organizados social, económica y políticamente, la percepción y las relaciones establecidas con la naturaleza, la definición y delimitación de territorios, entre otros, hacen parte de esta historia (Guerrero, 2004).

El trabajo del campesino, como cualquier proceso productivo por simple que sea, está soportado en un conjunto de ideas, conocimientos, valores, definiciones y creencias que interactúan con una estructura productiva específica (Argueta et al., 2011). Según la UNESCO (2003), salvaguardar una visión del mundo o un sistema de creencias es aún más difícil que preservar un entorno natural.

Además de las amenazas externas que se ciernen sobre su entorno social y natural, las comunidades campesinas propenden a adoptar modos de vida o modelos de 
desarrollo puramente económicos ajenos a sus tradiciones o costumbres. La protección del entorno natural está vinculada con la salvaguardia de la cosmología de una comunidad y de los elementos de su patrimonio cultural inmaterial (UNESCO, 2016).

Los saberes campesinos están en riesgo de perderse, por lo que su preservación significa asegurar su transmisión intergeneracional dentro de las comunidades, así como la protección de los conocimientos a los cambios socioeconómicos y culturales, en particular en términos del mantenimiento de su funcionalidad y su conexión dinámica con las prácticas de individuos y grupos, en su interacción diaria con su entorno (Oviedo et al., 2007).

Soto (2006), señala que la identidad no es otra cosa que aquello que nos diferencia de los otros tanto en el ámbito individual como colectivo. Supone la conciencia de pertenencia a un grupo determinado y una simbología propia que reclama ser reconocida, protegida y promovida.

La valoración social de determinadas propiedades de los ecosistemas y su preservación por parte de grupos campesinos e indígenas ha constituido y constituye aún, una parte fundamental del compromiso práctico de los campesinos con las condiciones físicas que posibilitan su modo de vida (Silvetti, 2011).

La Serranía de los Yariguíes ubicada en el departamento de Santander (Colombia), se identifica como un sistema orográfico que en virtud de sus características físicoclimáticas, régimen y localización se constituye en el remanente boscoso más conservado y de mayor proporción de Santander, el cual presenta vegetación de subpáramo y páramo. Es necesario mantener sus coberturas vegetales, ya que regulan la oferta hídrica de la región y el suministro de las cuencas hidrogeográficas: río Suárez, río Sogamoso y río Magdalena (del Ministerio de Ambiente, Vivienda y Desarrollo Territorial, 2005).

Razón por la cual, se debe realizar una correcta utilización del suelo rural, que incluya la concertación y la participación de la población de la microcuenca la Cinco Mil, con el fin de identificar y generar estrategias que permitan ordenar, planificar y regular el uso y manejo de los recursos naturales y las actividades económicas.

Al fortalecer la participación y recuperación de la identidad de la población se promueve un adecuado manejo de la Serranía y el desarrollo de esfuerzos de conservación complementarios. Mecanismos que incentiven a los actores la adopción y rescate de prácticas sostenibles de conservación.

Por consiguiente, los saberes de los campesinos de la microcuenca la Cinco Mil ubicada en la Serranía de los Yariguíes, representan una estrategia para conservar áreas de importancia ecológica y ecosistémica. El valorar, restaurar y proteger el patrimonio cultural es un indicador claro de la recuperación, reinvención y apropiación de una identidad cultural (Molano, 2006).

El interés de este estudio fue caracterizar los saberes campesinos que constituyen una estrategia para la conservación de la Serranía de los Yariguíes (Santander, Colombia), con el fin de fortalecer los procesos de apropiación cultural en la comunidad de la microcuenca la Cinco Mil. Reconociendo el valor que tiene la 
tradición oral como patrimonio cultural intangible de la Humanidad de manera que redunden en propuestas de sostenibilidad social, agrícola y ambiental.

\section{Metodología}

El estudio se basó en la investigación participativa, donde se combinan el conocimiento local y el científico para encontrar soluciones a los problemas locales, sacando el máximo de provecho posible a las oportunidades y recursos con que se cuenta (Casado y Mielgo, 2007).

La investigación participativa con agricultores representa una alternativa de integración y acercamiento entre el conocimiento tradicional y el conocimiento científico convencional; desde este punto de vista se plantean modos de relación entre agricultores y profesionales del campo, a partir del diálogo de saberes y del reconocimiento y respeto por el saber tradicional de las comunidades rurales y la forma de relación con la naturaleza (San Martín, 2002).

\subsection{Fases de trabajo}

1.Acercamiento al área de estudio (observación): reconocimiento de la realidad en cuanto al uso de los recursos naturales agrícolas y su relación con las prácticas campesinas y la cosmovisión del grupo. Visitas efectuadas a los municipios del Hato y Simacota desde agosto de 2016 hasta febrero de 2017

2.Aplicación de instrumentos participativos: se realizaron entrevistas semiestructuras a la población ubicada en las áreas protegidas de la microcuenca la Cinco mil. Se efectuó un muestreo no probabilístico intencional, en el cual se seleccionaron pobladores ubicados hace más de 40 años en el área rural de las veredas Montuosa de Simacota y Vega de San Juan y Hoya Negra en el Hato, ya que son las veredas que tienen jurisdicción en las dos áreas naturales protegidas de estudio.

3. Sistematización de la información: se analizó, transcribió y describió la información recopilada en las entrevistas. El análisis cualitativo se basó en el material discursivo y descriptivo.

\section{2 Área de estudio}

Esta investigación se realizó en la microcuenca la Cinco Mil, la cual se ubica en la cuenca del Río Suárez, en los municipios del Hato y Simacota, con un área de $18.140,90$, de las cuales el $41,91 \%$ que son $7.602,86$ ha, se encuentra dentro del Parque Nacional Natural Serranía de los Yariguíes y el 58,09 \% que son 10.538,04 ha restantes en el Distrito Regional de Manejo Integrado de los Yariguíes, en el departamento de Santander (Colombia) (Figura 1). 
Figura 1. Ubicación geográfica de la investigación

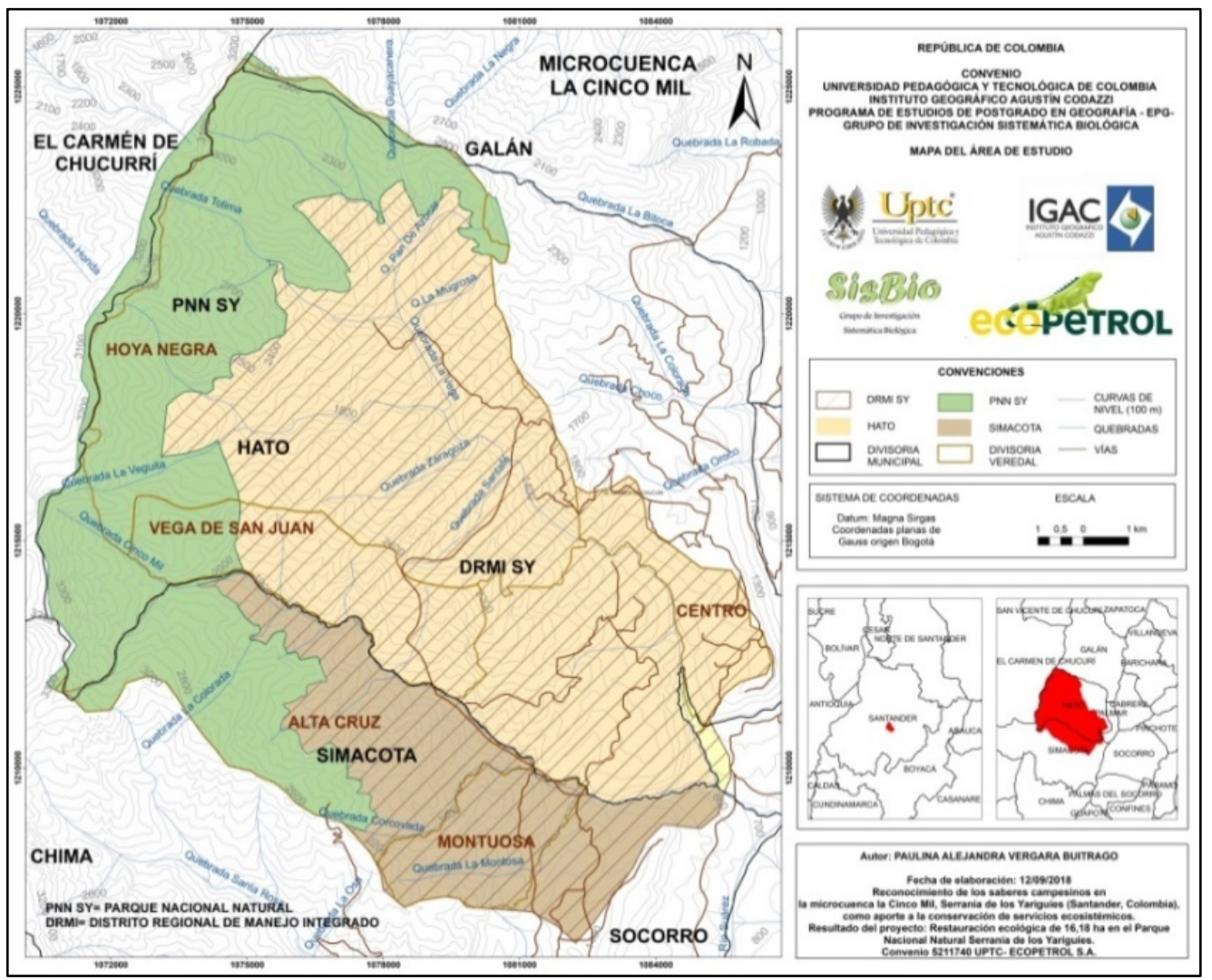

Fuente: Elaboración propia con base en el trabajo de realizado.

La zona del Hato y Simacota en la vertiente Oriental, son zonas de naturaleza secas con bajas precipitaciones (Interconexión Eléctrica S.A., 2002), lo que conlleva a que en estas áreas se presenten otras relaciones entre la vegetación y el sustrato. Según Duarte (2011), los asentamientos poblacionales han sido tradicionales debido a que su colonización estuvo ligada a un clima más sano que el de otras partes de la Serranía de los Yariguíes.

La agricultura es la principal actividad realizada en la región, representada en la producción de cultivos de café, yuca, arracacha, maíz, cítricos (naranja, mandarina) y plátano; además de labores de recolecta de productos y trabajos hogareños; asimismo la actividad pecuaria de ganadería de ceba, levante y crianza de ganado cebú, pardo surco y big master se realiza (Vergara, 2018). 


\section{Marco referencial sobre saberes campesinos}

Según Medina (1994) el campesino se define como el individuo o sujeto que labora y vive en el campo, que trabaja la tierra con su familia y que representa una cultura y un conjunto de valores concretos, que produce para recrear la familia y la unidad de producción. El campesino es el resultado histórico de distintos asentamientos humanos que van conformando grupos con identidades culturales que los caracterizan como sociedades (Ministerio de Agricultura y Desarrollo Rural, 1995).

Es la persona que habita y trabaja, sola o con su familia, en un territorio, dentro de una cultura, en estrecha interrelación con los actores sociales del mundo globalizado en la provisión de alimentos frescos, productos agroindustriales y artesanales en presencia de servicios ecosistémicos, aprovechando los biodiversos recursos naturales y culturales de su entorno (Núñez, 2001).

Los saberes campesinos son construcciones colectivas y dinámicas sociales que ayudan a organizar y dinamizar los quehaceres del campesinado en el sector rural. Afianzan la vida en el campo, generan unidad y potencian la representación grupal, tanto en sus siembras, en sus historias, como en la vida cotidiana (Arias, 2012).

Los saberes que surgen de la experiencia y que con el tiempo se van convirtiendo en saber local con el cúmulo social de cogniciones apropiadas y relativamente compartidas, que permiten a los integrantes de una cultura enfrentar los desafíos que les propone su medio ambiente. Estos saberes son transmitidos de generación en generación, por medio de la tradición oral (Gómez y Gómez, 2006).

En otras palabras, es importante el reconocimiento de la cultura campesina del País y de la necesidad de proteger su acceso a la tierra y al territorio, y con ello, su forma de vida culturalmente diferenciada. Dicho reconocimiento trasciende la formalización de títulos y enaltece la labor de los campesinos/as como fundamental en el desarrollo del País.

\section{Resultados y discusión}

Lo humano sólo existe como tal si se realiza en la pluralidad de sus versiones concretas, cada una de ellas distinta de las otras, cada una sui generis. Anular esa diversidad equivaldría a la muerte de lo humano. Felizmente, esa homogeneización es imposible: el mapa de la diversidad humana nunca perderá la infinita multiplicidad de su colorido. La diferencia es inevitable. No hay fuerza que pueda uniformar el panorama abigarrado de las identidades humanas (Echeverría, 2010).

La vitalidad de las comunidades, por no decir de las comunidades apartadas de las fuerzas desarrollistas del modelo precapitalista, depende de forma directa de la conservación de los recursos naturales, que contribuyen a dar forma a su estilo de vida, su cultura, su interrelación, su crecimiento y a la continuidad física de la comunidad (Melgar, 2001).

A partir del trabajo realizado en campo con la comunidad campesina de los municipios del Hato y Simacota (Santander), se propiciaron encuentros participativos, 
donde se compartieron conocimientos entre los diferentes miembros de la comunidad, logrando así identificar información valiosa oral de las costumbres que se han venido transmitiendo de generación en generación.

Es común que los miembros más viejos de las comunidades posean mejores conocimientos y más detallados que los jóvenes (Tapia, 2002). Los conocimientos sobre prácticas agrícolas se han generado por enseñanzas transmitidas de padres a hijos; la vida en familia era unida, se participaban en la labor de siembra de productos como: café, yuca, maíz, arracacha, cítricos, plátano, entre otros; realizando uno o dos cosechas al año en épocas de lluvia.

El sector agropecuario se ha constituido en la principal ocupación productiva de las familias en Santander, es por ello que el $95 \%$ de sus habitantes sustentan sus ingresos con las labores del campo, el producto de la actividad agrícola en su mayoría es utilizado para su propia subsistencia, del cual consume el $40 \%$ de lo cosechado (Plan de Desarrollo Municipal, 2016).

La preparación manual de suelos con arado de caballos y bueyes, incorporando abonos verdes aumenta la disponibilidad de materia orgánica y mejora la estructura del suelo. El cultivo se establece cerca de vegetación nativa, con asociaciones incluyendo especies como las forrajeras y aromáticas. Así mismo, se incorporan animales al sistema de producción que representen un beneficio frente al aprovechamiento de estiércoles para la elaboración de abonos.

Al emplear este tipo de arado el suelo no esta tan compactado, ya que el peso del animal es inferior al del tractor. Además, en el terreno se presenta una cobertura protectora, ya que no se entierran totalmente los rastrojos, manteniéndose así las capacidades del suelo, que contribuyen la producción agrícola. El manejo de tractor incide de manera negativa sobre las características físicas del suelo, como el aumento de la compactación y la exposición de los microorganismos del suelo a la radiación solar, cuando los arados de disco voltean el suelo.

Antes las familias sembraban en conjunto, desde los abuelos hasta los nietos; se araba por medio de la yunta en bueyes y se picaba con azadón o rastrillo. Según Tamayo (2013), las técnicas agrícolas tradicionales implicaban la utilización de remedios contra plagas, fabricados en forma casera a base de plantas locales, la preparación del terreno y la utilización de herramientas como la yunta o el azadón.

En la actualidad la población joven no está interesada en permanecer en el área rural cultivando, debido a la falta de mejores oportunidades de vida, lo cual ha generado que la gente adulta migre al área rural dejando tierras productivas de siembra abandonadas.

Los habitantes entrevistados manifiestan que en el proceso de fertilización del suelo, que se realizaba hace 20 años, se empleaban elementos naturales como: cal, ceniza, excrementos de los animales (guano) y el tamo del trigo y la cebada; para el control de plagas y enfermedades en los cultivos utilizaban ají, barbasco, ceniza, cal y sulfato; las malezas eran extraídas manualmente del suelo o utilizando herramientas como el hoz, acciones que se desarrollan actualmente en las huertas orgánicas que algunos han establecido en sus fincas. 
Al elaborar abonos orgánicos, así como, caldos orgánicos, líquidos foliares a base de estiércol de bovinos enriquecidos con sales minerales y melaza; se generan productos, para controlar las deficiencias de micronutrientes en suelos desgastados, mejoramiento de la fertilidad del suelo, ataque de plagas y enfermedades en los cultivos presentes en las fincas.

Para que las plantas de los cultivos se desarrollen vigorosas y se obtengan buenas cosechas hay que aprender a identificar cuáles son los nutrientes que se encuentran en la tierra de la parcela, cuáles son los que faltan, que clase de nutrientes aportan los abonos y cómo y cuándo deben aplicarse. Según Vergara et al., (2018) la fertilidad del suelo puede mejorar con los cuidados necesarios y una buena aportación de abonos orgánicos.

Un elemento importante para destacar dentro de los sistemas de producción campesina es su afinidad con la producción agroecológica en la mayor parte de éstos, el cual representa baja dependencia de insumos externos para actividades agropecuarias como la labranza, siembra, cultivo, riego, deshierbe, abonamiento y control de arvenses, una gran diversificación dentro de la producción agropecuaria, con multiplicidad de productos y uso de los diferentes estratos del suelo (Santocoloma, 2015).

Una parcela debe sembrarse alternando cultivos diferentes, así el terreno se aprovecha mejor y la familia obtiene para su consumo varios productos. Se tenía la costumbre de realizar rotación de cultivos transitorios, como el maíz o yuca, intercalados con zonas de café; el agua para riego era obtenida de lluvia o por escorrentía- gravedad; las semillas para cultivar se conseguían del intercambio de semillas con vecinos o de las cosechas que iban saliendo, al momento de sacar las plantas se identificada la mejor para obtener semillas de esta.

La diversidad incorporada en los sistemas de cultivo provee de nutrientes al cultivo e interrumpe el ciclo de vida de varios insectos, plagas, enfermedades y malezas. La rotación de cultivos ayuda al control de problemas fitosanitarios, al evitar la presencia de los mismos patógenos en los cultivos.

La mejor selección de semilla que se puede hacer es desde la mata, al momento de la colecta se va seleccionando la semilla y se va echando a una vasija o costal, para sacar la semilla que sea pareja, y se clasifica desde el arranque. De acuerdo con Suárez et al., (2007), el jefe era el que hacía este trabajo, indicándole a un obrero cuáles semillas se seleccionaban.

La agricultura campesina, desde sus inicios hace miles de años, se ha dedicado a seleccionar, mejorar, obtener, guardar, multiplicar e intercambiar semillas libremente. Según Vinueza (2014), la transmisión libre de saberes y de conocimientos, a partir de las experiencias de toda persona agricultora, ha marcado las relaciones humanas con la madre tierra, proveedora y dadora de alimentos y de vida y ha contribuido en la difusión de las bases de la actual biodiversidad agrícola.

La eliminación manual de hierbas arvenses consiste en sacar de raíz con la mano las hierbas invasoras. Se emplea en casos donde la hierba tiene alta capacidad de reproducción vegetativa. Asimismo; en el corte manual, se utilizan herramientas 
como machetes, azadón y pala. La eliminación manual es la más sostenible, pero a su vez la más laboriosa de los tipos de control de hierbas.

La aplicación de productos químicos, en especial herbicidas, traen consigo diferentes desventajas como: la disposición de envases, contaminación del suelo y agua, son tóxicos para los seres vivos, el uso indistinto afecta las coberturas naturales, esterilización de los suelos, entre otras.

Para la siembra los campesinos esperaban a que hubiera luna creciente, mientras que en luna menguante se realizaba la poda o corte; nunca se sembraba cuando el suelo estaba muy húmedo o seco, y se acostumbraba a nombrar a San Isidro Labrador para que se diera una cosecha productiva, así como colocar una cruz entre los cultivos en el mes de mayo en honor a la Santísima Virgen. Según Vásquez et al., (2015), el conocimiento tradicional que tiene el productor sobre los efectos de la luna en la producción agrícola y pecuaria son prácticas muy antiguas que han adquirido de sus ancestros.

Durante la recolección de la cosecha los campesinos tenían la tradición de hacer un almuerzo de integración, donde se daba gracias a San Isidro por la cosecha ofreciéndole una limosna, además de coronar al patrón. Una parte de la cosecha se comercializaba llevándola en burro o mula a las plazas de mercado más cercano, el resto era para el consumo de la familia. Según Calderón et al., (2004), San Isidro es la deidad referida tradicionalmente a la actividad agrícola (como intermediario en los ciclos de lluvia y sol).

La preservación de los saberes campesinos significa asegurar su transmisión dentro de las comunidades y apoyar la adaptación de los conocimientos a los cambios socioeconómicos y culturales, en particular en términos de sostenimiento de su funcionalidad y su conexión dinámica con las prácticas de individuos y grupos en su interacción diaria con su entorno (Yumicasa, 2013).

En lo relacionado con los ecosistemas, los saberes campesinos tienen aplicación en un modelo de agricultura sostenible. Desde tiempos atávicos y como herencia, los antepasados hacían un uso racional del suelo, sin utilizar productos de síntesis química y desarrollando sistemas de cultivo orgánicos, rotación de cultivos, asociación de cultivos, entre otros.

Además de ser una propuesta de producción de alimentos sanos, se convierte en una estrategia para evitar el deterioro ambiental que sufren los ecosistemas andinos actualmente. En el ámbito social, se evidencia que la tradición oral fomenta los valores de la familia, la convivencia humana en comunidad, el trabajo colectivo y una visión del mundo más humana.

Las prácticas y creencias en el uso y conservación de productos autóctonos están seriamente amenazadas por los contextos institucionales, que no salvaguardan de forma efectiva y directa la protección de estas prácticas de cultivo. Además, del modelo global de desarrollo, que otorga limitados espacios en el mercado a la agricultura tradicional, por lo general, ya marginada (Clavijo y Pérez, 2014).

Según Valdebenito (2005), entender el territorio no sólo como un espacio sobre el cual transcurre la vida social, sino un artífice de esa realidad; un territorio históricamente construido en el cual ocurren y se han desarrollado determinados 
procesos sociales y culturales permite la activación social del patrimonio cultural; que transforma la identidad y supone un reconocimiento y apropiación de la memoria histórica, del pasado. Un pasado que puede ser reconstruido o reinventado, pero que es conocido y apropiado por todos.

Hacer geografía es preocuparse por interpretar el mundo actual, es indagar sobre las relaciones entre el medio y el hombre, y explicar cómo se reparten estas relaciones diversas en la superficie terrestre. Lo demás es reflexionar, calentarse las mentes sin sentido, en suma, hacer lo que no es geografía (Olcina, 1997).

Es imposible comprender cómo se han desarrollado las diferentes relaciones sociales en la tierra, si no se realiza una revisión histórica, economía y política de los fenómenos que han sucedido y como estos han influenciado en la transformación del entorno, logrando así tomar conciencia de la relación que siempre ha existido entre el hombre y su entorno.

El medio ambiente es un sistema, del cual el hombre forma parte integradora y no es sólo un eslabón, al discutir la relación del ser humano con el medio ambiente se debe comprender, que el medio ambiente se ha convertido en una construcción social, en donde cada acción desarrollada influye positiva o negativamente; generar conciencia sobre el cambio ambiental que se está presentando, es un reto que todos han de afrontar.

Los procesos investigativos deben partir de la realidad y necesidades de las comunidades, ejecutándose de manera que reconozcan la gente, su historia, memorias y conocimiento del territorio. Idealmente la investigación debe ser asumida con los habitantes locales, e incluso por ellos mismos, con orientación adecuada si fuera el caso; buscando que puedan manejarse en marcos de autonomía y enfocarse sobre lo propio, indagando sobre el bienestar, reconocimiento y permanencia de la comunidad (Riveros et al., 2015).

Los procesos de desarrollo sostenible, al tomar lugar en el territorio, requieren ajustarse a las condiciones del espacio natural, así el territorio podrá adecuarse para facilitar dicho desarrollo, a través de un ordenamiento territorial, cuidando que se garantice la sostenibilidad y equidad.

Cada entidad territorial posee su propia escala espacial y de gobierno (municipal, departamental, nacional), teniendo en cuenta que su organización territorial es interdependiente y que las estructuras espaciales que soportan los diversos procesos de desarrollo son sistémicas; es decir, en conjunto se interrelacionan y complementan e idealmente deberán converger alrededor del objetivo común de alcanzar un desarrollo y ordenamiento territorial equilibrado y sostenible (Secretaría Técnica COT, 2013).

La comunidad utiliza la especie forestal nativa denominada como aro (Trichanthera gigantea), para la protección de quebradas; ya que tiene afinidad con el agua, un rápido crecimiento y ayuda en el control de la erosión, asimismo la materia orgánica que genera aporta nutrientes al suelo.

La siembra de árboles nativos alrededor de las fuentes hídricas permite controlar la erosión de las mismas y la supervivencia de especies animales que dependen de los frutos que generan los bosques. El conocimiento de los campesinos sobre la 
vegetación nativa constituye una herramienta que se debe emplear para recuperar cuencas hidrográficas y coberturas antropizadas. La naturaleza necesita del trabajo de todos.

La conservación y con ella la sostenibilidad territorial, debe encontrar su expresión más concreta en el ordenamiento territorial, pues mediante esta herramienta políticoadministrativa se orientan y planifican acciones físicas concertadas, para orientar el desarrollo del territorio y para regular la utilización, transformación y ocupación del espacio, de acuerdo con las estrategias de desarrollo socioeconómico y en armonía con el medio ambiente y las tradiciones históricas y culturales (Ley 388, 1997).

Según la comunidad el suelo ha sido la base para los árboles y los bosques. Es un elemento importante de los bosques, ya que regula procesos ecosistémicos, como la descomposición de materia orgánica, la filtración de nutrientes, y la disponibilidad de agua. Las prácticas de gestión del suelo, que incluyen acciones para conservar la cubierta de los suelos, contribuyen a reducir la pérdida de la fertilidad en éstos.

Para salvaguardar los suelos se deben proteger los árboles. Estos recursos realizan una función primordial para la salud del medio ambiente. La cobertura arbórea, la vegetación, y los bosques previenen la degradación del suelo en la Serranía de los Yariguíes.

Como señala Unwin (1995), cuando nos pregunten a qué nos dedicamos, debemos ser capaces de decir con confianza que somos geógrafos; que nos ocupamos de algunas de las cuestiones más vitales que se plantean en la sociedad contemporánea. Una geografía con sentido global, donde se muestre al hombre en relación con el medio y la sociedad en la que vive.

La teoría de las representaciones sociales estudia el proceso por el cual los grupos humanos construyen conocimientos del sentido común en torno a objetos sociales específicos, lo que le permite integrar un conjunto de conceptos tradicionalmente utilizados por la psicología social como actitudes, opiniones, creencias y estereotipos, entre otros (Jodelet, 1986).

Se observa aquí una interesante similitud entre representaciones sociales y los saberes campesinos, ya que ambas conceptualizaciones ponen su foco de interés en los conocimientos del sentido común de los sujetos, es decir, en aquellos que les permiten dar sentido a su experiencia y orientar sus comportamientos en la vida cotidiana (Landini, 2010).

En este sentido, al reconocer los saberes de los campesinos de la microcuenca la Cinco Mil, se identificaron representaciones sociales de la población. Se destaca la importancia de apoyar y fomentar los procesos de autoaprendizaje y la circulación de conocimientos generados al interior de las comunidades, con el fin de potenciar el uso de los saberes apropiados. Según González (2007), el estudio de los saberes campesinos vistos como representaciones sociales permite analizar aspectos puntuales que dan sentido a las prácticas de las agrícolas desarrolladas por los campesinos.

A partir de los instrumentos participativos aplicados, se identificaron los siguientes saberes campesinos (Tabla1), como estrategias que promueven la conservación de los servicios que brindan los ecosistemas localizados en la Serranía de los Yariguíes, así como al desarrollo rural sostenible de la región. 
Tabla 1. Saberes de los campesinos de la Serranía de los Yariguíes

\begin{tabular}{|c|c|}
\hline COMPONENTE & SABERES CAMPESINOS \\
\hline \multirow{4}{*}{ Agrícola } & Desarrollo de arado con caballos y bueyes \\
\hline & $\begin{array}{c}\text { Rotación de cultivos: Variación en las siembras para mejorar la } \\
\text { riqueza del suelo }\end{array}$ \\
\hline & Aprovechamiento de residuos orgánicos \\
\hline & Eliminación manual de hierbas arvenses \\
\hline \multirow[b]{2}{*}{ Natural } & Siembra de árboles nativos alrededor de las fuentes hídricas \\
\hline & $\begin{array}{l}\text { Mantener las coberturas arbóreas de la Serranía de los Yariguíes, ya } \\
\text { que protegen los suelos }\end{array}$ \\
\hline \multirow{2}{*}{ Cultural } & Astrológicas: ciclos de la luna \\
\hline & Festividades: Celebración a San Isidro Labrador \\
\hline
\end{tabular}

Fuente: Elaboración propia a partir del trabajo de campo realizado.

Figura 2. Representación del agroecosistema del área de estudio.

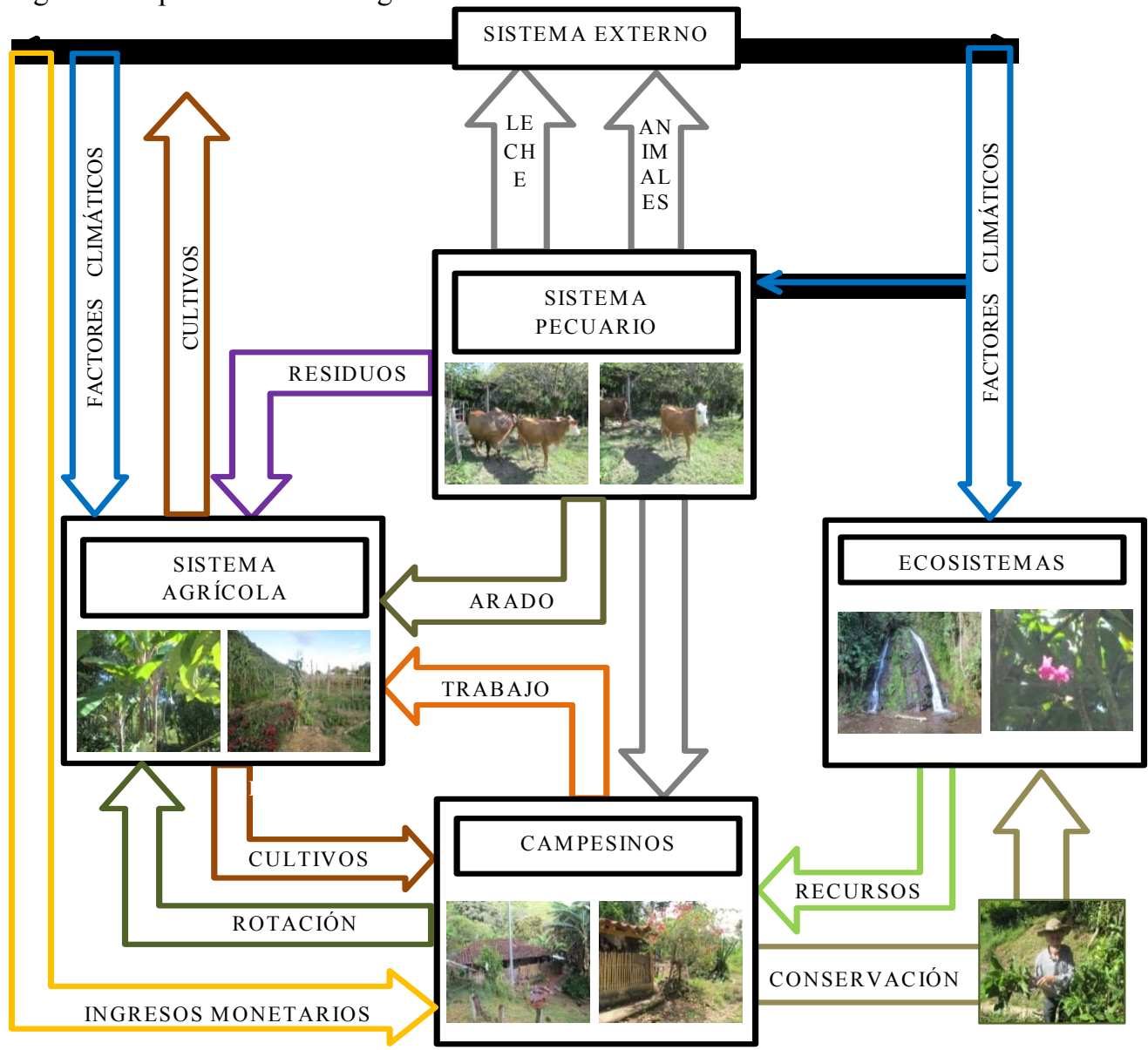

Fuente: Elaboración propia con base en el trabajo de realizado. 
Los saberes campesinos desarrollados por la comunidad de Yariguíes se constituyen en buenas prácticas agrícolas, las cuales se definen como un conjunto de principios, normas y recomendaciones, que se aplican a las diversas etapas de la producción agrícola para garantizar principalmente al abastecimiento de alimentos sanos e inocuos. En la figura 2 se observa la articulación de los saberes campesinos en las laborares desarrolladas al interior de los agroecosistemas, los cuales contribuyen a la conservación de la microcuenca la Cinco Mil.

Se identificaron cuatro componentes (campesinos, ecosistemas, sistema agrícola y sistema pecuario), los cuales se articulan con el desarrollo de los saberes campesinos reconocidos, en especial con la actividad de agrícola que realizan los campesinos, en la cual emplea materias primas que obtienen de la Serranía de los Yariguíes y a su vez generan acciones para conservar los recursos que reciben.

\section{Conclusiones}

La formación del campesino colombiano tiene una historia de la cual el hombre rural de hoy es su directo heredero. La autenticidad de sus tradiciones proviene de, mantener viva su cultura y los vínculos que lo atan a su trabajo y de mantener con firmeza su sistema de valores sociales y religiosos. El cultivador, el agricultor, el artesano, el pescador, el recolector de Norte a Sur, de Oriente a Occidente, ocupa el territorio patrio con su actividad incesante (Mejía, 1989).

Al identificar los saberes de los campesinos de la microcuenca la Cinco Mil, se reconocieron buenas prácticas agrícolas como el desarrollo de arado con caballos y bueyes, rotación de cultivos, aprovechamiento de residuos orgánicos, eliminación manual de hierbas arvenses, siembra de árboles nativos alrededor de las fuentes hídricas y mantenimiento de coberturas arbóreas, que constituyen estrategias para la protección de los servicios ecosistémicos de la Serranía de los Yariguíes.

Los saberes campesinos representan una categoría de análisis, para estudiar la agricultura como una forma de vida, en virtud de que para los campesinos los conocimientos no están apartados de las creencias o valores, por el contrario, estos conocimientos son formas de conducta y acciones prácticas que los campesinos desarrollan como parte de su diario vivir, esta identidad campesina se materializa en la apropiación de los servicios ecosistémicos.

El territorio permite una identidad, en la misma medida que el hombre interactúa con el medio, y genera un vínculo de relaciones y la apropiación colectiva de cada una de las manifestaciones culturales que se desarrollan en los territorios, razón por lo cual se deben proveer diferentes escenarios que permitan a los diversos grupos sociales que existen, compartir cada uno de sus conocimientos y experiencias, con el fin de generar una conciencia y existencia del individuo y de su entorno.

El reconocimiento de los saberes campesinos se convierte en una estrategia para replicar en otras comunidades. Donde el acercamiento a los niños y jóvenes debe ser promovido, con el fin de generar un centro de intercambio de experiencias con los adultos y ancianos de las comunidades. Los conocimientos rurales permiten su 
aplicación para resolver problemas ambientales y para promover la conservación de los servicios ecosistémicos.

Los resultados de este trabajo aportan información para futuros estudios que se adelanten con la comunidad rural ubicada la microcuenca la Cinco Mil; en las cuales, las iniciativas de ordenación, planificación y regulación del uso de los recursos naturales deben ir articuladas con el conocimiento de la población frente a las actividades económicas que se realizan allí, con el propósito de promover la conservación de servicios ecosistémicos.

La cultura es la memoria del pueblo, la conciencia colectiva de la continuidad histórica, el modo de pensar y de vivir (Kundera, 1992).

\section{Referencias bibliográficas}

Argueta, A; Corona, E y Hersch, P. (2011): Saberes colectivos y diálogo de saberes en México. México: Ibero Puebla.

Arias, J. (2012): Educación rural y saberes campesinos en Tierradentro Cauca: estudio del proceso organizativo de la Asociación Campesina de Inzá Tierradentro (ACIT). (Tesis doctoral), Universidad Nacional de Colombia.

Calderón, L; Pereira, L; Chirinos, O y López, I. (2004): Diagnóstico sociocultural Ceuta Tomoporo: Antropología del desarrollo para la industria petrolera. Boletín Antropológico., $\mathrm{N}^{\circ} 58$, p. $165-186$.

Casado, G y Mielgo, A. (2007): La investigación participativa en agroecología: una herramienta para el desarrollo sustentable. Revista Ecosistemas. $\mathrm{N}^{\circ} 16(1)$, p. 24-36.

Clavijo, N y Pérez, M. (2014): Tubérculos andinos y conocimiento agrícola local en comunidades rurales de Ecuador y Colombia. Cuadernos de Desarrollo Rural. $\mathrm{N}^{\circ} 11(74), \mathrm{p}$. 149-166.

Duarte, I. (2011): Diagnóstico de los aspectos físico-bióticos del Parque Nacional Natural Serranía de los Yariguíes, Santander-Colombia. San Vicente de Chucurí - Colombia.

Echeverría, B. (2010): Modernidad y blanquitud. México: Ediciones Era.

Gómez, J y Gómez, J. (2006): Saberes tradicionales agrícolas indígenas y campesinos: rescate, sistematización e incorporación a las IEAS. Ra Ximhai. № 2(1), p. 97-126.

González, M. (2007). Agroecología y agricultura como forma de vida. Revista Brasileira de Agroecología. $\mathrm{N}^{\circ}$ 2(2), p. 415-418.

Guerrero, A. (2004): La santandereanidad: un debate en construcción. Revista Pretextos., N ${ }^{\circ}$ 16 , p. $17-18$.

Interconexión Electrica S.A (2002): Prioridades de Conservación de la Biodiversidad de la Serranía de los Yariguíes en Jurisdicción de la CAS. Plan de Manejo ambiental para la línea de Transmisión eléctrica Primavera-Guatiguará-Tasajero.

Jodelet, D. (1986): La representación social: fenómenos, concepto y teoría. En: Moscovici, S. Pensamiento.

Kundera, M. (1992): La insoportable levedad del ser. México: Editores RBA. 
Landini, F. (2010). La dinámica de los saberes locales y el proceso de localización del saber científico. Algunos aportes desde un estudio de caso. Cuadernos de Desarrollo Rural. $\mathrm{N}^{\circ} 7$, (65), p. 21-43

Ley 388. (1997): Santafé de Bogotá: Ministerio de Desarrollo Económico. Viceministerio de Vivienda, Desarrollo Urbano y Agua Potable.

Medina, J. (1994): Nuevas formas de organización y producción comunitaria. Caso las Lajitas, Sanare, Estado Lara: Universidad Central de Venezuela, CENDES.

Mejía, M. (1989): Colombia campesina. Santafé de Bogotá: Villegas Editores.

Melgar, A. (2001): Perspectivas rurales $\mathrm{N}^{\circ}$ 2, ambiente, desarrollo rural y seguridad alimentaria, comportamiento y contexto sociocultural comunitario que influye sobre el ambiente y los recursos naturales en el departamento de jalapa Guatemala. Costa Rica: Imprenta IICA. p. 144.

Ministerio de Agricultura y Desarrollo Rural. (1995): Censo de minifundio en Colombia. Santafé de Bogotá: Impreandes Presenica S.A.

Ministerio de Ambiente, Vivienda y Desarrollo Territorial. (2005): Resolución 603 de mayo de 2005. Se declara el Parque Nacional Natural Serranía de los Yariguíes. Publicado en el Diario Oficial No 46.004, del 18 de agosto de 2005. Colombia.

Molano, O. (2006). Identidad cultural un concepto que evoluciona. Revista Opera. № 7, p. 6984.

Núñez, J. (2004). Los saberes campesinos: implicaciones para una educación rural. Investigación y Postgrado. $\mathrm{N}^{\circ} 19(2)$, p. 13-60.

Olcina, J. (1997): La Geografía hoy: Reflexiones sobre el pensamiento geográfico, la región y la docencia de la Geografía. Investigaciones geográficas. $N^{\circ} 16$, p. 93-114.

Oviedo, G; Noejovich, F y Zamudio, T. (2007): Desafíos para el mantenimiento de los conocimientos tradicionales en América Latina. En: Resumen Ejecutivo del Informe solicitado por la Conferencia de las Partes del Convenio sobre Diversidad Biológica.

Plan de desarrollo municipal. (2016): Alcaldía de Hato (2016-2019). Disponible en internet: http://www.hato-santander.gov.co/Nuestros planes.shtml?apc=gbxx-1-\&x=2460737

Riveros, A; Cuellar, C; Ríos, C; Rodríguez, C, Castillo, R, Hortúa, C; Clavijo, C; Polindara, C y Díaz, C. (2015): Los páramos y su gente: Agenda ciudadana para un territorio posible. BogotáD.C.: Tropenbos Internacional Colombia \& UICN Sur.

San Martín, J. (2002): Participación de los actores en la implementación de proyectos de desarrollo. Memorias del curso taller: metodologías participativas: hacia el diálogo de saberes. Cochabamba. Maela.

Santocoloma, L. (2015): Importancia de la economía campesina en los contextos contemporáneos: una mirada al caso colombiano. Entramado. $\mathrm{N}^{\circ} 11(2)$, p. 38-50.

Secretaría Técnica COT. (2013): Lineamientos para el proceso de ordenamiento territorial departamental contenidos básicos. Departamento Nacional de Planeación.

Silvetti, F. (2011): Una revisión conceptual sobre la relación entre campesinos y servicios ecosistémicos. Cuadernos de desarrollo rural. $\mathrm{N}^{\circ} 8(66)$, p. 19-45.

Soto, D. (2006): La identidad cultural y el desarrollo territorial rural, una aproximación desde Colombia. RIMISP Territorios con identidad cultural.

Suárez, R; Peña, D y Amaya, A. (2007): Cultivando papa en complicidad con la naturaleza. Revista Luna Azul. $\mathrm{N}^{\circ} 24$, p. 23-34. 
Tamayo, A. (2013): La agricultura orgánica y la agricultura tradicional: una alternativa intercultural. Letras Verdes. Revista Latinoamericana de Estudios Socioambientales. $\mathrm{N}^{\circ} 4$, p. 24-26.

Tapia, N. (2002): Agroecología y agricultura campesina sostenible en los Andes bolivianos: el caso del ayllu Majasaya Mujlli, Departamento de Cochabamba. Bolivia: Plural editores.

UNESCO. (2003): Convención para la salvaguardia del patrimonio cultural inmaterial. Disponible en Internet: http://www.portal.unesco.org/es/

UNESCO. (2016): Conocimientos y usos relacionados con la naturaleza y el universo. Disponible en Internet: https://ich.unesco.org/es/conocimientos-relacionados-con-lanaturaleza-00056

Unwin, T. (1995): El lugar de la Geografía. Madrid: Editorial Cátedra.

Valdebenito, R. (2005): Identidades territoriales y patrimonio cultural: la apropiación del patrimonio mundial en los espacios urbanos locales. Revista teórica del Departamento de Ciencias de la Comunicación. $\mathrm{N}^{\circ} 1$, p. 289-306.

Vásquez, A; Narváez, J y Calero, W. (2015): Los efectos de la luna en la producción agropecuaria. Revista Universitaria del Caribe. $\mathrm{N}^{\circ} 13(2)$, p. 21-25.

Vergara, P. (2018). Reconocimiento territorial participativo de la Serranía de los Yariguíes, microcuenca la Cinco Mil, (Santander-Colombia). Revista de Investigación Agraria y Ambiental. $\mathrm{N}^{\circ}$ 9(2), p. 217-230.

Vergara, P; Morales, M; Useche, D y Gil, P. (2018): Encuentros para el reconocimiento y aprendizaje ambiental con la comunidad campesina del páramo de Rabanal, Boyacá, Colombia. Revista Geográfica Venezolana. N 59(2). 398-410.

Vinueza, S. (2014): La semilla: patrimonio de los pueblos al servicio de la humanidad. Universidad de Barcelona.

Yumicasa, S. (2013): Erosión de conocimientos ancestrales de los productores de papa en cinco comunidades de la UCASAJ, parroquia San Juan, Cantón. Riobamba, provincia de Chimborazo. Disponible en internet: http://dspace.ups.edu.ec/bitstream/123456789/6406/1/UPS-QT40911.pdf

Zaccagnini, M. (2014): Manual de buenas prácticas para la conservación del suelo, la biodiversidad y sus servicios ecosistémicos área piloto Aldea Santa María, Entre Ríos. Argentina: Programa de las Naciones Unidas para el Desarrollo. Instituto Nacional de Tecnología Agropecuaria. 